\title{
La transparente opacité du masque ironique. Bronzino à l'épreuve de l'ironie figurative
}

\section{Pauline Martin}

\section{(2) OpenEdition}

1 Journals

Édition électronique

URL : http://journals.openedition.org/imagesrevues/320

DOI : 10.4000/imagesrevues.320

ISSN : 1778-3801

Éditeur:

Centre d'Histoire et Théorie des Arts, Groupe d'Anthropologie Historique de l'Occident Médiéval, Laboratoire d'Anthropologie Sociale, UMR 8210 Anthropologie et Histoire des Mondes Antiques

\section{Référence électronique}

Pauline Martin, «La transparente opacité du masque ironique. Bronzino à l'épreuve de l'ironie figurative », Images Re-vues [En ligne], 1 | 2005, document 1, mis en ligne le 01 septembre 2005, consulté le 31 janvier 2021. URL : http://journals.openedition.org/imagesrevues/320 ; DOI : https:// doi.org/10.4000/imagesrevues.320

Ce document a été généré automatiquement le 31 janvier 2021.

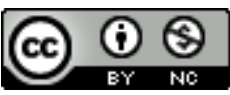

Images Re-vues est mise à disposition selon les termes de la Licence Creative Commons Attribution Pas d'Utilisation Commerciale 4.0 International. 


\title{
La transparente opacité du masque ironique. Bronzino à l'épreuve de l'ironie figurative
}

\author{
Pauline Martin
}

1 Le paradoxe de l'ironie picturale ne se réduit pas seulement au phénomène figuratif que cet article propose de décrire. Il demeure aussi dans le puits, petit, mais sans fond dans lequel tombe chaque recherche parue sur le sujet. L'absence d'étude sur l'ironie picturale, mis à part dans le cadre de l'art contemporain qui place souvent l'ironie au cœur de sa démarche, est en effet

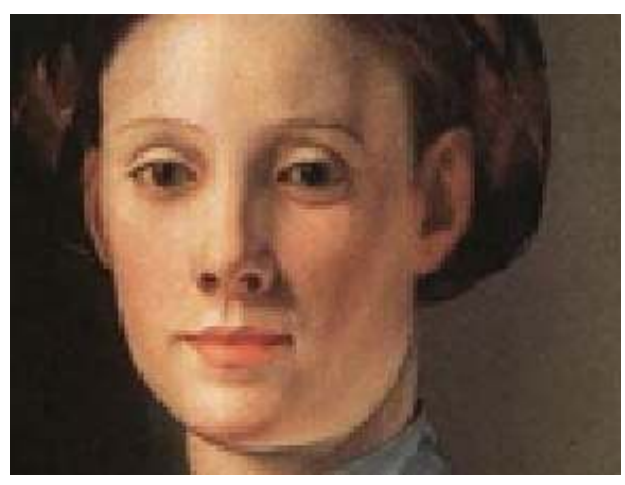
déplorée par la plupart des historiens qui

s'y intéressent, alors même qu'ils contribuent à combler ce vide. Il est en effet étonnant d'observer que les recherches de Paul Barolsky et d'Ernest B. Gilman dans les années 1970, qui abordent de manière plus large l'humour dans l'art du XV $\mathrm{Xu} \mathrm{XVII}^{\mathrm{e}}$ siècles, puis les divers articles qui s'intéressent à l'ironie particulière d'une œuvre ou d'un artiste, ne se sont pas du tout accumulés pour faciliter la compréhension de l'ironie dans la peinture figurative; ils se sont le plus souvent juxtaposés sans prendre en considération les contributions précédentes ${ }^{1}$.

Ce constat suggère deux hypothèses. Premièrement, l'étude historique de l'ironie figurative nécessite une indexation très précise sur le corpus choisi. Non seulement l'ironie est une notion historique dont les composantes changent, mais sa dimension intentionnelle, subjective et inévitablement contextuelle empêche aussi à son étude d'avoir une valeur d'emblée générale. Un tel calibrage contextuel explique sans doute en partie que les articles monographiques ne débouchent jamais sur une généralisation quelconque de l'ironie picturale. 
3 L'absence d'approche théorique de l'ironie figurative constitue le second obstacle à l'émergence d'un domaine de recherche reconnu comme tel. Dans la plupart des cas, le choix du corpus et l'intérêt historique porté à celui-ci précèdent le désir d'appréhender le phénomène de l'ironie figurative de manière théorique. Les tentatives d'une compréhension $\mathrm{du}$ dispositif représentatif mis en jeu dans l'ironie restent très modestes et se limitent généralement à une phrase, au mieux un paragraphe. Les descriptions de l'ironie picturale et figurative se bornent ainsi à la considérer comme une opposition de signes contradictoires (Barbara Budnick ${ }^{2}$ ) ou comme une distance autoréférentielle de l'œuvre sur elle-même (James Elkins'). Aucune d'entre elles ne paraît toutefois véritablement satisfaisante, si on les compare aux théories abondantes que la linguistique a consacrées au phénomène discursif de l'ironie.

\section{De l'ironie verbale à l'ironie picturale}

4 Ce foisonnement dans la discipline de la linguistique, bien que stimulant et indispensable, constitue paradoxalement l'une des entraves à l'élaboration d'une théorie de l'ironie figurative. Il ne s'agit pas ici de transposer la théorie linguistique à l'analyse de la peinture - projet illusoire qui risquerait de dénaturer la particularité figurative -, mais bien de décrire au mieux le dispositif de représentation qui donne à voir une ambiguïté ironique. La théorie linguistique est donc à la fois indispensable pour comprendre la notion même d'ironie, mais pourtant malcommode dans la mesure où les innombrables définitions, terminologies et nuances qui ont été données de l'ironie depuis l'étude fondatrice de Dan Sperber et Deirndre Wilson sont adaptées à la description du discours et non de l'image ${ }^{4}$. Afin de mieux m'en détacher par la suite, je résumerai donc ici d'emblée les acquis théoriques essentiels de la linguistique, car ils seront à l'arrière-plan de ma tentative de description de l'ironie picturale.

5 Il convient d'abord de rappeler que la définition antiphrastique de l'ironie (qui consiste à «faire entendre le contraire de ce qu'on dit $»^{5}$ ) a été déboutée dès la fin des années 1970 au profit de compréhensions plus nuancées qui ont l'avantage, du point de vue de l'historien de l'art, de lui éviter de croire qu'une image ironique montrerait le contraire de ce qu'elle représente. Depuis Oswald Ducrot, en particulier, l'ironie est considérée comme un phénomène énonciatif et plus spécifiquement comme une forme particulière de polyphonie : «L'énoncé signale, dans son énonciation, la superposition de plusieurs voix $»^{6}$. Premier point : l'ironie ne consiste pas à transmettre un message unique et caché en explicitant son contraire, mais bien à juxtaposer et à faire exister, en même temps, deux sens différents. Deuxième élément : cette coexistence de plusieurs sens est signalée dans l'énonciation, dans la manière dont l'énoncé est formulé et exécuté ; c'est donc la forme du message ironique qui indique la pluralité des points de vue exprimés. Troisième point important : les significations de l'énoncé ne doivent pas seulement être multiples, elles doivent surtout entrer en conflit pour instituer une polarisation et une mise à distance ${ }^{7}$. L'ironie joue sur plusieurs niveaux de langage, qui se fondent l'un dans l'autre tout en s'opposant et se superposant. Au premier degré de l'énoncé, qui correspond au sens littéral, s'ajoute donc un deuxième degré, qui commente et met à distance, sans pour autant le rejeter, ce qui est explicitement dit.

Ces quelques éléments théoriques, posés ici de manière schématique et beaucoup trop succincte, font partie des outils qui ont permis à l'analyse littéraire d'accorder une place primordiale à l'ironie. Le phénomène est aujourd'hui connu: l'ironie est une 
catégorie dans laquelle ont été rangés de nombreux textes ainsi que certains de leurs auteurs ; c'est devenu l'un des traits constitutifs de la littérature moderne, voire de la littérarité. Il n'en est pas de même dans l'histoire de la peinture, qui caractérise parfois de manière un peu légère et anecdotique certaines œuvres comme ironiques, mais presque sans jamais considérer cette qualité comme première et essentielle à la compréhension de l'image. C'est sans doute que, dans sa forme figurative, l'ironie n'est pas assez bien définie et lisible, et qu'elle ne peut par conséquent pas être repérée comme une catégorie à part entière. Le phénomène se situant aux frontières d'autres registres, notamment le sérieux, le comique, le sarcasme ou la parodie, il est souvent plus aisé pour les historiens de l'art de classer les œuvres dans ceux-ci que dans celui de l'ironie ${ }^{8}$. Elle constitue pourtant bien un genre en soi, « elle n'est pas "neutre", mais littéralement tragi-comique; l'un-et-l'autre plutôt que ni-l'un-ni-l'autre " ${ }^{9}$, comme l'a très bien montré Jankélévitch. Il s'agit donc ici de problématiser les questions qui entrent en jeu dans l'analyse de l'ironie figurative et de proposer quelques repères pour s'approcher d'une compréhension plus stable du phénomène.

\section{L'ironie en discussion}

Fig. 1

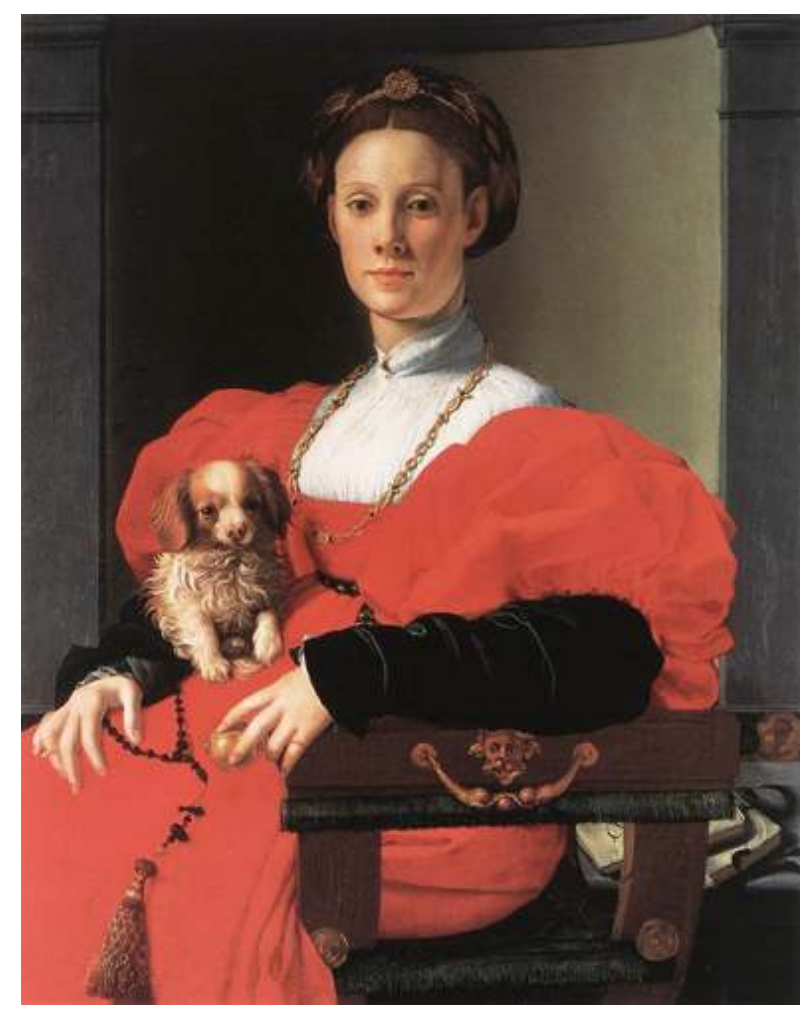

Agnolo Bronzino, Portrait de dame au petit chien, 1532-1536, huile sur bois, 90 x 71 cm, Städelsches Kunstinstitut, Frankfurt am Main. Avec l'aimable autorisation du musée.

www.staedelmuseum.de 
Fig. 2

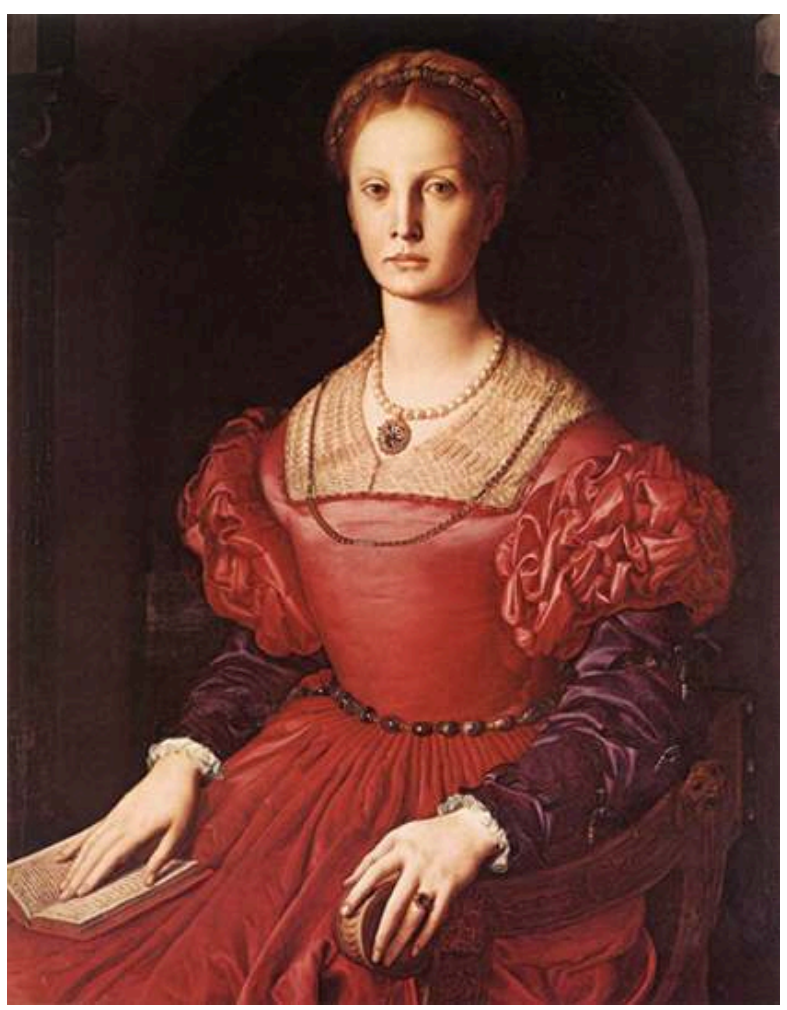

Agnolo Bronzino,Portrait de Lucrezia Panciatichi, vers 1540, huile sur bois, $102 \times 85$ cm, Galleria degli Uffizi, Firenze. Avec l'aimable autorisation du Ministero dei Beni e le Attività Culturali.

www.polomuseale.firenze.it/uffizi

Quatre portraits peints par Bronzino entre 1530 et 1555 serviront de support analytique pour dégager quelques principes importants de l'ironie figurative, tels qu'ils me sont apparus dans l'état actuel de ma recherche. Le Portrait de dame au petit chien (fig. 1), daté par les historiens du début des années 1530, le Portrait de Lucrezia Panciatichi (fig. 2), le Portrait du jeune homme au livre (fig. 3), probablement effectués tout deux vers 1540 et le Portrait de dame (fig. 4), conservé à Turin et daté de la première moitié des années 1550 , ont tous la particularité d'être accompagnés, à l'intérieur du cadre du tableau, de grotesques qui ornent l'architecture ou les meubles autour des modèles. Si le motif du grotesque et les compositions particulières des œuvres, qui permettent de mettre en avant quelques principes théoriques, ont en grande partie motivé ce choix de cas pratiques, c'est avant tout une raison historiographique qui a présidé à sa sélection. Plusieurs historiens ont en effet émis des avis contrastés au sujet du caractère ironique de ces œuvres. Par l'opposition des poses sérieuses et des grotesques grimaçants, les portraits éveillent intuitivement, bien qu'anachroniquement, un soupçon d'ironie qui a été tantôt défendu, tantôt rejeté. Ces interprétations, opposées sur le point qui m'intéresse, montrent de manière exemplaire que l'ironie picturale se laisse difficilement saisir, et qu'elle est bien souvent appréhendée à partir de catégories connexes qui accentuent une seule de ses composantes à la fois. 
Fig. 3

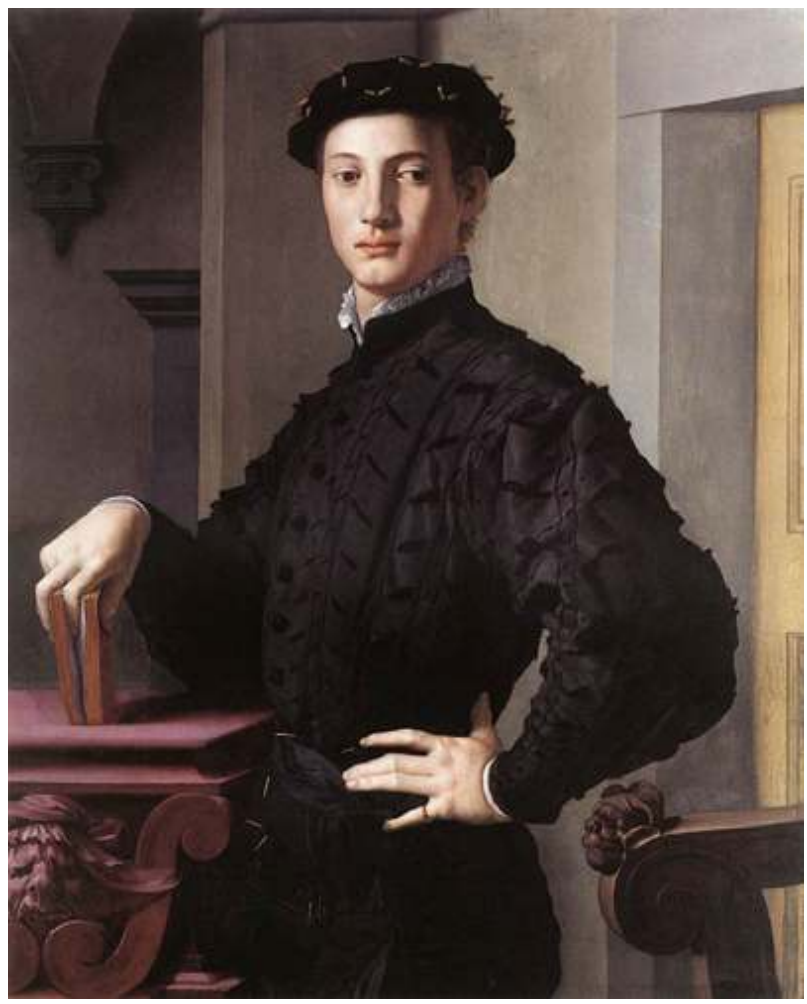

Agnolo Bronzino, Portrait de jeune homme au livre, vers 1540, huile sur bois, 96 × $75 \mathrm{~cm}$, Metropolitan Museum of Art, New York.

www.metmuseum.org 
Fig. 4

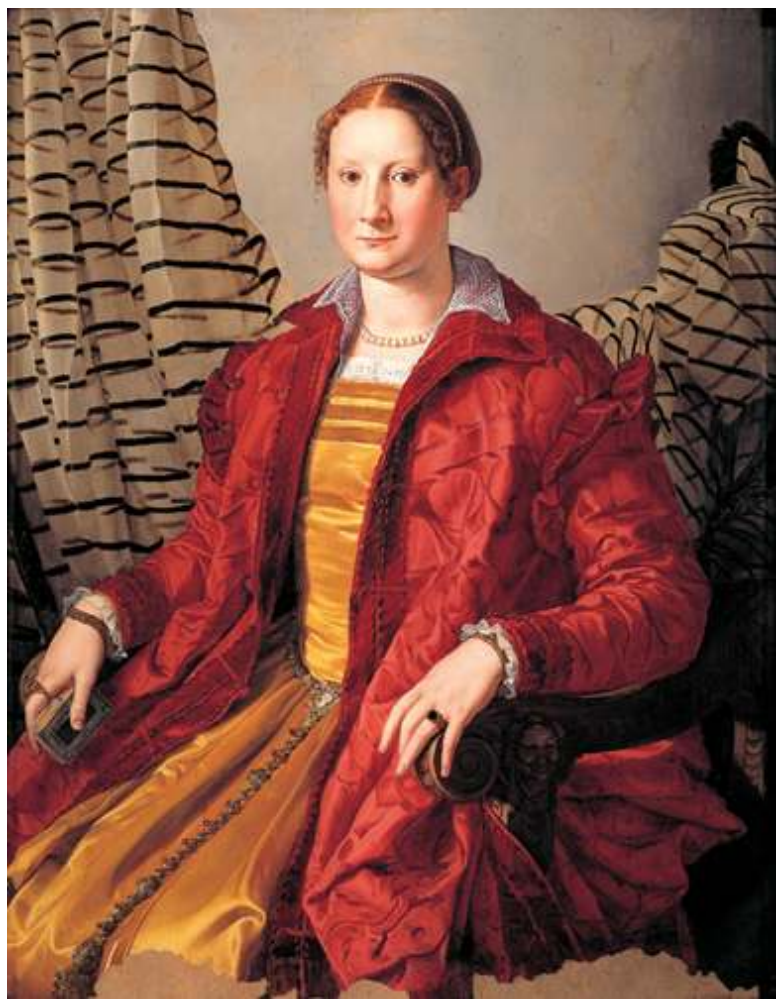

Agnolo Bronzino, Portrait de dame, vers 1555, huile sur bois, $109 \times 85 \mathrm{~cm}$, Galleria Sabauda, Torino Avec l'aimable autorisation du Ministero dei Beni e le Attività Culturali.

www.artito.arti.beniculturali.it

Dans son étude sur la production littéraire de Bronzino, Deborah Parker estime, au sujet des grotesques sur la balustrade et sur le fauteuil près du jeune homme au livre, que « les grotesques fonctionnent comme un demi-sourire ironique méditant sur une telle démonstration de la conscience de sa propre élégance $»^{10}$. De même, Paul Barolsky affirme que «ces éléments grossiers et bizarres suggèrent une lucidité ironique du courtisan par rapport à lui-même: il pose de manière très élégante, tout à la fois conscient et en même temps distant de ces éléments ${ }^{11}$. Survol utile mais rapide de nombreux aspects comiques dans les œuvres italiennes des $\mathrm{XV}^{\mathrm{e}}$ et $\mathrm{XVI}^{\mathrm{e}}$ siècles, l'étude de Barolsky ne donne aucune précision sur les conditions qui légitimeraient, à Florence au milieu du $\mathrm{XVI}^{\mathrm{e}}$ siècle, la présence d'une telle ironie, dont il ne définit ni le mécanisme, ni la nature.

A l'opposé de Barolsky, Maurice Brock, auteur de la monographie la plus récente parue sur Bronzino, donne une analyse très sérieuse des portraits qui nous intéressent - j'y reviendrai plus en détail par la suite. Il faut pourtant souligner ici la nécessité qui s'impose au spécialiste de discuter la valeur comique des grotesques pour l'atténuer. S'il admet qu'«il n'est pas totalement exclu que [...] l'invention abondante dans les moindres choses que Bronzino développe à partir du motif du mascaron vise à amuser le spectateur» et qu'«il se peut aussi qu'avec leur hybridité cocasse, ces motifs introduisent dans le portrait une touche de gaieté qui atténue le sérieux de la pose et la gravité de l'expression $»^{12}$, le comique n'a selon lui pas sa place chez Bronzino dans une œuvre par ailleurs sérieuse ; il rejette le mélange des genres dans une seule œuvre, qui est une source fondamentale de l'ironie: «Le peintre ne mélange 
qu'exceptionnellement les genres: en règle générale, il les distingue aussi soigneusement que le poète. [...] Dans les portraits de cour, rien ne prouve que les braguettes emphatiques ou les mascarons grimaçants qui ornent les meubles aient alors produit un effet comique $»^{13}$.

\section{Vers une description théorique de l'ironie picturale}

10 Ces interprétations des portraits de Bronzino, insistant tantôt sur leur dimension ironique, tantôt sur leur sérieux, ne sont pas nécessairement opposées. Chacune d'entre elles va d'ailleurs servir mon analyse. Aux deux degrés de l'ironie correspondent en effet deux regards différents, bien que simultanés, portés sur les portraits. Le premier niveau - que l'on pourrait qualifier de littéral - ne prend en considération que l'image du monde feinte dans la plus pure transitivité de l'œuvre; il considère l'image explicite qui transparaît dans l'illusion de sa tridimensionnalité. Puisqu'il constitue le message le plus immédiatement compréhensible des images, c'est par celui-ci que va s'ouvrir la description. Les portraits qui nous intéressent expriment au premier regard le sérieux et la noblesse de leurs commanditaires. L'idéalisation des modèles, dont le visage acquiert une géométrie presque parfaite, et leurs expressions corporelles - leurs gestes suggérés, leurs poses, leurs expressions faciales ainsi que leurs vêtements - indiquent leur appartenance au monde de la cour et désignent leur rang social élevé. Elles répondent à la codification du paraître qui se met en place à la Renaissance, et dont le Livre du courtisan de Castiglione, paru en 1528, témoigne en détail ${ }^{14}$.

11 Au XVIe siècle, les grotesques situés sur le mobilier et l'architecture qui entourent les modèles constituent une deuxième affirmation de leur adhésion aux valeurs courtisanes. Ces éléments étaient en effet en totale adéquation avec le monde de la cour auquel les modèles appartenaient. A Florence, au XVIe siècle, cet ornement décoratif était très apprécié, non seulement dans les grands programmes picturaux ${ }^{15}$, mais également dans les arts décoratifs ${ }^{16}$. Les grotesques, sur le mobilier, indiquent ainsi le goût très à la mode des personnages portraiturés. Dans le Portrait de Lucrezia Panciatichi (fig. 2) ainsi que dans celui du Jeune homme au livre (fig. 3), mais avec une certitude moindre en raison du manque d'informations sur l'identité du modèle, les mascarons sont également le signe de leur sensibilité à la littérature et à l'humour burlesque largement apprécié et valorisé par la cour des Médicis $^{17}$. Principalement littéraire, le burlesque avait également une forme picturale. Le Nain Morgante de Bronzino, effectué pour Cosme Ier, son corps difforme et obèse, ses jambes courtes, son nez recourbé et sa coupe de vin à la main, en est une des manifestations les plus évidentes. Le grotesque, bien sûr, en est une autre. Selon Philippe Morel, la poésie burlesque s'offre en partie à une comparaison avec le grotesque ${ }^{18}$. Sans faire entrer les portraits dans le registre burlesque (ils n'annulent en effet pas tout le sérieux de la pose qui domine), les grotesques font référence à ce genre particulier. En accolant ces visages déformés aux portraits, le peintre annonce le goût des modèles pour cet humour de la cour, tout en affirmant leur noblesse par leur pose. Les têtes déformées sur le bas du Portrait du jeune homme au livre (fig. 3) forment ainsi avec le livre qu'il tient entre les mains un indice significatif de l'appartenance du courtisan au cercle littéraire que fréquentait Bronzino, et qui appréciait largement l'humour burlesque. Les deux grotesques sur le siège de Lucrezia Panciatichi (fig. 2), née Pulci et épouse de Bartolomeo Panciatichi qui était 
comme Bronzino membre de l'Accademia Fiorentina, dès 1541, sont quant à eux une réminiscence de Luigi Pulci, ancêtre du modèle et auteur de Morgante le Géant en 1483, écrit précisément dans ce style burlesque qu'allait faire connaître Francesco Berni.

Les grotesques, en plus de constituer un signe des valeurs courtisanes des commanditaires, servent surtout à renforcer leur idéalisation. Maurice Brock a montré comment les mascarons, physiquement soumis aux modèles, mettent en valeur, par contraste, la beauté des visages humains: «Le mascaron grimaçant suggère que l'inexpressivité du visage humain résulte du processus de civilisation des mœurs. Le mascaron symbolise les vices, les pulsions débridées de l'animalité. Le visage tendant à l'impassibilité symbolise les vertus, le raffinement par la socialisation des pulsions $»^{19}$ (19). Selon lui, les portraits présentent une "progression ascendante de l'animalité grimaçante à l'humanité policée $\aleph^{20}$. Les têtes sculptées et déformées ne sont pas uniquement décoratives; elles ont également une fonction de faire-valoir par la comparaison qu'elles instaurent avec les visages idéalisés des modèles. Cette progression, qui passe directement des grotesques aux figures idéalisées dans le Portrait de Lucrezia Panciatichi (fig. 2) et dans le Portrait du jeune homme au livre (fig. 3), se fait en trois temps dans les deux autres portraits qui nous intéressent. Dans le Portrait de dame au petit chien (fig. 1), entre l'animalité dépravée des mascarons et la sociabilité idéalisée du modèle, se situe, à mi-hauteur, un animal domestique, comme un palier entre la sauvagerie et l'inexpressivité raffinée ${ }^{21}$. C'est une face humaine sculptée sur la ceinture de la dame qui constitue, dans le portrait de Turin, le degré intermédiaire de réalité et d'expressivité entre le mascaron et le modèle ${ }^{22}$. Prenant appui sur les pendentifs de la voûte consacrée aux vertus cardinales que Bronzino effectue en 1541 dans la chapelle d'Eléonore de Tolède et qui montrent des masques sous les représentations des vertus, Maurice Brock donne une signification unique aux grotesques : «l'agencement vertical [...] assigne aux masques un sens univoque: en application du traditionnel principe figuratif de superposition qui marque le triomphe du vainqueur sur le vaincu ou la domination du bien sur le mal, les masques symbolisent les vices sur lesquels les vertus l'emportent. La symbolique est vraisemblablement diffuse au sens où chaque masque ne représente sans doute pas un vice précis. [...] Le vice est collectivement présenté sous des dehors laids ou risibles $»^{23}$.

Cette interprétation univoque des grotesques ne permet pourtant pas d'épuiser toutes leurs ressources, dans la mesure où elle n'insiste pas assez sur leur artificialité picturale. Par l'importance qu'elle donne à la composition verticale des œuvres et à l'idéalisation artificielle des modèles, cette première description des portraits met l'accent sur le fait que les visages humains ne nous apparaissent pas dans la réalité, mais bien sur une toile entièrement maîtrisée par le peintre. En revanche, elle n'accorde pas le même traitement aux grotesques qui sont considérés pour ce qu'ils représentent et non pour la manière dont ils se présentent au spectateur: l'interprétation se base sur ce que les mascarons sont, collectivement, et non sur leurs configurations particulières et sur la façon dont chaque grotesque est modelé sur la toile. Et c'est bien le fait qu'ils sont des grotesques difformes et laids par nature qui importe, et non leurs traits particuliers ${ }^{24}$. Les mascarons d'un portrait pourraient alors remplacer ceux d'un autre sans que cette permutation n'en bouleverse le sens. C'est qu'ils sont considérés dans la "transitivité de la représentation ${ }^{25}$ dont la théorie critique a été proposée par Louis Marin, et qui n'apparaît que lorsque la matérialité et l'artificialité des signes picturaux s'effacent derrière ce qu'ils signifient du réel. 

transitive. Au XVI ${ }^{e}$ siècle, leur sens n'était pas strictement établi et ils réclamaient souvent une explication qui en résolve l'énigme ${ }^{26}$. Par la rupture qu'ils introduisent dans des situations tout entières dévolues à l'expression du sérieux et de la noblesse, les grotesques signalent l'existence d'un sens dissimulé et conçu par Bronzino. Ils se présentent comme un indice de l'importance de l'intervention du peintre. Ils permettent au spectateur de dépasser la dimension transitive de l'œuvre pour se souvenir de l'artificialité de la scène qui lui est présentée et de la responsabilité de l'artiste. Pour reprendre les termes de Louis Marin, les mascarons provoquent « une discrète rupture de la "transitivité blanche" de la représentation $»^{27}$. L'étude de Daniel Arasse sur le détail permet d'ajouter que "regardé pour lui-même, le détail iconique fait surgir l'opacité insensée de la peinture " en "déplaçant l'attention du sujet représenté à la manière dont il est représenté $\aleph^{28}$. Du représenté émerge la perception de la représentation. Par ce nouveau regard posé sur le grotesque, où importe moins la signification du signe que son apparence, la question "qu'est-ce qui est représenté » laisse place à une nouvelle interrogation : « comment est-ce représenté ?». œuvres et de la mise en comparaison des mascarons et des visages humains. Elle doit se poursuivre sur la forme spécifique de chaque mascaron, et sur la manière dont ils évoluent par rapport aux portraits particuliers auxquels ils sont rattachés. Cet aspect est essentiel, puisqu'il ne limite pas le grotesque à un symbole diffus du vice, mais insiste plutôt sur son individualisation en fonction des modèles. C'est de cette observation des traits spécifiques de chaque mascaron, et de la manière particulière dont ils sont imbriqués à chaque modèle, qu'émerge l'ironie.

Dans le Portrait de dame au petit chien (fig. 1), du début des années 1530, la tête de la femme est très légèrement tournée vers la gauche, alors que celle du chien est pour sa part inclinée vers la droite. Par leurs positions, le modèle et l'animal se complètent et affichent une frontalité dont ils constituent chacun un pendant. Celle-ci répond au mascaron qui nous fait face. Selon Brock, « leur coprésence et leur frontalité amènent inévitablement à les comparer $»^{29}$. Cette comparaison révèle que les traits du grotesque sont déterminés par les deux têtes qui le dominent : sa bouche et son nez s'apparentent à ceux d'un chien alors que les oreilles, à moitié humaines, tombent également comme celles de l'animal. Le mascaron assimile la femme et le chien non seulement par sa frontalité, qui répond à la réunion des positions du modèle et de l'animal, mais aussi par ses traits mi-canins mi-humains.

17 Cette hybridité disparaît entièrement dans le grotesque du Portrait de dame de Turin (fig. 4), pour s'apparenter au nouveau modèle auquel il est comparé. Aucun animal n'accompagnant la dame, il n'adopte cette fois que des traits humains. Ceux-ci répondent à la physionomie de la femme sur trois points. D'abord, la forme arrondie du mascaron correspond à celle du visage humain. Ensuite, les figures présentent toutes deux un front dégarni. De plus, le large sourire du grotesque fait un écho chargé à celui, plus timide, de la dame. Il est en effet important de remarquer que des quatre portraits qui nous intéressent, celui-ci est le seul à présenter un modèle souriant; il exhibe également l'unique mascaron riant.

Lucrezia Panciatichi, dans son portrait (fig. 2), adopte au contraire une expression très sérieuse. La bouche tombante et énervée du mascaron que frôle sa main s'y accorde. La manière dont le grotesque est mis en parallèle avec le modèle révèle par ailleurs la 
volonté de les relier fermement l'un à l'autre. Le fauteuil dans lequel la femme est assise comporte un accoudoir extrêmement sophistiqué qui se compose uniquement de courbes. Les éléments qui le relient au siège sont en effet arrondis, de même que le morceau principal sur lequel la modèle pose son bras gauche. Ainsi, le grotesque du fond nous apparaît de trois quarts alors que celui que Lucrezia touche de sa main nous fait face de manière très frontale. Ses yeux croisent directement ceux de l'observateur, comme le font ceux de Lucrezia Panciatichi. Le grotesque, ainsi lié au visage du modèle, perd en outre la possibilité d'être une simple décoration de meuble, dans la mesure où, très bizarrement, le deuxième accoudoir est absent. Derrière le modèle, sur la partie gauche du tableau, le fauteuil semble comme évanoui: ni accoudoir, qui devrait pourtant se laisser deviner derrière la main qui tient le livre, ni prolongement $\mathrm{du}$ dossier qui donnerait ainsi l'idée d'un banc. Le meuble perd sa fonction première de fauteuil, et prend celle de support au grotesque, dans le but de l'opposer au visage de la femme.

19 Dans le Portrait du jeune homme au livre (fig. 3), c'est à la fois le cadrage de l'image, sa mise en scène et l'apparence caractérisée des deux grotesques qui les nouent au modèle. Le triangle, formé par la posture de sa tête et de ses bras, se prolonge dans les deux grotesques sur le bas du tableau. Ces derniers, intégrés par cette composition très simple à la figure du jeune homme, le sont encore plus par le cadrage et par la mise en scène des objets choisis par Bronzino. En réponse à la pose de trois quarts du visage du jeune homme, qui dissimule ainsi son oreille droite, le grotesque sur la balustrade cache aussi la sienne par le cadre du tableau qui la coupe. Le grotesque de profil, en revanche, exhibe son oreille gauche, étonnamment grande et mise en valeur, en parallèle à celle du courtisan qui nous est présentée. En outre, de même que le Portrait de dame de Turin (fig. 1) est le seul à afficher un sourire sur le mascaron et le modèle, le Portait du jeune homme au livre (fig. 3) présente pour sa part l'unique modèle portant un chapeau auquel correspondent les seuls grotesques avec des couvre-chefs.

\section{La « transparente opacité du masque ironique »}

Observés au niveau réflexif et opaque de l'image, les grotesques révèlent l'ironie qu'ils engendrent. Individualisés en fonction des personnages qu'ils accompagnent, imbriqués aux visages humains par les particularités de représentation et de composition des œuvres, les mascarons ne peuvent se réduire univoquement au vice vaincu par la socialisation des modèles. A cette fonction valorisante, qui existe bien réellement dans les portraits, s'en ajoute une deuxième dévalorisante, qui, sans l'annuler, entre en contradiction avec la première. En effet, les grotesques ne se laissent pas totalement dominer par les visages humains policés parce qu'à leur tour, par leurs traits individualisés et caricaturaux, ils imposent leurs accents aux modèles ${ }^{30}$. Par l'écho chargé qu'ils leur font, les mascarons - et ainsi le vice et la laideur qu'ils désignent - se présentent comme une part négative inhérente aux personnages représentés. Si les grotesques sont le vice et la laideur que les dames et le jeune homme ont vaincus par l'apparence et le masque social, ils sont également le vice individualisé, - et incorporés à chacun d'entre eux. Les modèles n'y échappent pas totalement, parce que les mascarons leurs sont fermement attachés. Au lieu de les détacher clairement les uns des autres, de manière à éviter toute confusion, Bronzino les rapproche, laissant la porte ouverte à l'ambiguïté et au «double voir », pour faire un parallèle au «double 
entendre » si fréquent dans sa littérature burlesque. Le grotesque n'a pas une fonction qui serait déterminée a priori; il n'est pas toujours porteur d'ironie ${ }^{31}$. Mais leur représentation particulière, dans chacun des quatre portraits de Bronzino, indique que leur sens n'est pas univoque, mais au contraire ambigu, paradoxal et ironique.

L'ironie figurative se joue donc sur deux niveaux parallèles, qui sont ici décortiqués de manière analytique, mais qui sont généralement expérimentés par le spectateur de manière spontanée et simultanée. D'un côté, le niveau transitif et transparent de l'image met en valeur le représenté, qui asseoit une première lecture dans l'illusion de la tridimensionnalité et de la réalité. D'un autre côté, le niveau intransitif et opaque de l'œuvre fait émerger, au-delà de sa matérialité, la représentation; il institue un deuxième degré de signification, en concurrence avec le premier sens déjà perçu et qui le met à distance. Ces deux significations différentes ne s'annulent pas l'une l'autre; elles se superposent au contraire de manière exacte, dans les traits de l'image, et sont simultanément et entièrement visibles à l'intérieur du cadre du tableau. Dans les portraits de Bronzino, les grotesques cristallisent tous ces enjeux. Leur premier sens la valorisation du modèle - se perçoit dans la profondeur feinte du représenté ; leur deuxième sens - la critique ou la moquerie du modèle - se marque dans cette surface plane de la représentation qui permet de modeler les grotesques en fonction des visages humains, et qui présente les mascarons comme une facette négative, et non plus uniquement positive, des modèles. C'est dans l'interaction de ces deux niveaux de représentation, à la fois visibles, parce que tous deux devant les yeux du spectateur, et invisibles, parce que se superposant l'un l'autre, que se situe «l'invisible visibilité, la transparente opacité du masque ironique ${ }^{32}$. L'ironie ne consiste donc pas simplement en une confrontation de signes contradictoires dans une même œuvre. L'opposition du sérieux des modèles de Bronzino et de la légèreté des grotesques qui les accompagnent n'épuise pas tout le dispositif de l'ironie figurative. Celle-ci n'existe qu'à la condition que cette opposition ne soit pas statique et dirigée vers un sens univoque; et c'est la réversibilité de l'interprétation des mascarons, constituée par le double niveau de représentation évoqué plus haut, qui est le réel garant de l'ironie. L'opposition de signes contradictoires ne constitue que l'un des indices possibles de l'ironie (et c'est le cas dans les portraits de Bronzino), mais qui n'est pas indispensable au dispositif ironique.

\section{De la théorie à l'histoire : à la recherche de l'intention ironique}

Telle qu'elle vient d'être faite, la description théorique d'un phénomène figuratif ne suffit pas à le prouver. Encore faut-il le justifier historiquement. Le phénomène est bien entendu avant tout affaire d'interprétation. Des éléments peuvent nous paraître aujourd'hui ironiques, qui ne l'étaient pas du tout au XVIe siècle. Ce n'est pourtant qu'en regardant les œuvres - acte que nous ne pouvons bien évidemment faire que dans notre temps - que nous pouvons espérer une compréhension, et avant même celle-ci, une intuition à leur sujet. Il s'agit ensuite de la valider par une approche historique, qui reste la seule valable dans une tentative de compréhension de la notion même d' "ironie picturale". Ou, pour le formuler autrement, Bronzino était-il capable de produire de l'ironie, et ses contemporains pouvaient-il la comprendre ? Ou encore : Bronzino a-t-il eu une intention ironique? Problématique dans le cadre d'une étude 
historique, la notion d'intention cristallise plusieurs questions que pose l'étude de l'ironie figurative. La disposition mentale et l'esprit du peintre étant irrévocablement perdus, il s'agit plutôt, dans une perspective historique, de s'approcher au plus près des motivations du peintre, qui, à près de cinq siècles de distance, nous parviennent sous différents aspects ${ }^{33}$. L'aperçu de l'ensemble de la production de l'artiste, d'abord dans la mesure où elle est un produit partiel de sa disposition d'esprit, permet d'appréhender ses capacités d'ironiste ${ }^{34}$. Le contexte historique, et plus particulièrement la place que tenait l'ironie à son époque, ainsi que la compréhension qu'on en avait, éclaire également l'intention du peintre. Celle-ci inclut enfin les raisons qu'il a pu avoir de s'exprimer de manière ironique dans son œuvre, ainsi que, dans le cadre de portraits de commande, les motivations des modèles.

C'est avant tout la disposition favorable de Bronzino envers l'ironie qu'il faut ici relever. Le mélange des registres à la fois sérieux et burlesque qui transparait de l'ensemble de son œuvre nous oblige à le considérer de manière très subtile: les portraits dynastiques qu'il effectue de la famille Mécidis, les fresques de la chapelle d'Eléonore de Tolède, Vénus et Cupidon de la National Gallery de Londres et son Nain Morgante révèlent un artiste dont l'esprit ne s'était pas rangé dans un seul répertoire et qui pouvait, comme l'exige l'ironie, allier le sérieux et le facétieux. Selon Deborah Parker, auteure de la seule étude sur la production littéraire du Florentin, «l'artistepoète ne choisit pas une voix contre une autre. En effet, il met souvent les deux formes en contact de manière amusante. Dans l'œuvre de Bronzino, le lyrique et le burlesque, le sacré et le profane, le sublime et le cru coexistent. Dans une mesure moindre mais néanmoins remarquable, la peinture de Bronzino présente la même double tendance créatrice. [...] La manière dont Bronzino s'empare simultanément de deux formes poétiques suggère qu'il pourrait être productif de considérer son œuvre moins comme impénétrable que comme imprégnée par deux formes d'expression, l'une ordonnée et élégante, l'autre désordonnée et subversive $\aleph^{35}$. La littérature de Bronzino, aussi importante et abondante que sa production picturale, signale également sa disposition pour l'ironie. Auteur de plus de 200 poèmes raffinés, il est aussi l'écrivain de 11 sonnets satiriques, et de plus de 30 longs capitoli, parfois obscènes et volontairement subversifs. Ecrits dans le registre burlesque, qui utilise fréquemment « l'éloge paradoxal qui fait porter aux nues un moustique ou une mouche $»^{36}$, ses capitoli confirment l'inclination de Bronzino pour l'ironie.

Ce trait esthétique, repérable dans son œuvre, doit être envisagé dans le contexte plus large de la Florence du XVIe siècle qui appréciait et pratiquait l'ironie, comme en témoignent les études de Dilwyn Knox et de Norman Knox ${ }^{37}$. L'ironie constituait en effet un trait d'esprit valorisé non seulement dans la littérature, mais aussi dans la conversation de la société courtisane. Dans le livre II qu'il consacre en partie à l'humour adapté à la vie de cour, Castiglione recommande ainsi « la sorte de facétie qui tient de l'ironie" non seulement aux courtisans, mais plus encore "aux grands personnages, parce qu'elle est grave et pleine de sel, et qu'on peut en user dans les choses joyeuses et aussi dans les choses sérieuses $»^{38}$. Il vante ainsi, à travers les mots de l'Aretin, l'«élégante manière de raillerie que celle qui consiste en une certaine dissimulation, quand on dit une chose et que tacitement l'on en entend un autre $»^{39}$. Au XVIe siècle, cette ironie se manifestait souvent par la relative dissimulation de sa propre valeur en raison de l'assimilation alors courante de l'ironie à l'ironie socratique $^{40}$. Redécouverte à la Renaissance dans les dialogues platoniciens, celle-ci 
consiste, pour l'époque, à se déprécier soi-même ironiquement, le plus souvent en feignant l'ignorance ${ }^{41}$. L'ironie, à la fois comme trait d'esprit et comme autodépréciation ironique socratique, était alors souvent associée à de l'auto-ironie, qui permettait d'exprimer une modestie dont on verra l'importance dans les portraits de Bronzino.

L'existence d'une ironie figurative au XVIe siècle florentin reste pourtant encore à établir. Si, à cette époque, l'ironie discursive est en effet connue et valorisée, quoiqu'il soit alors important de " considérer avec soin la limite et la mesure à respecter quand on fait rire de façon piquante, et qui est celui que l'on pique $»^{42}$, elle reste cantonnée à la langue, du moins dans les différents textes qui la thématisent. Le mécanisme décrit par Castiglione relève de la conversation et non de la représentation. Cette distinction est fondamentale. La problématique générale de cet article consiste précisément à tenter une compréhension de l'ironie figurative, qui ne peut se réduire à une simple comparaison avec le discours. Un tel dispositif pouvait-il être mis en place par un peintre? En l'absence de tout document écrit de l'époque, qui expliciterait et validerait une telle démarche, les œuvres contemporaines de Bronzino peuvent conforter l'idée que la peinture pouvait penser de la sorte au temps du maniérisme. C'est en particulier une peinture de Vasari, effectuée à Florence vers 1535, ainsi que l'analyse qu'en fait Edouard Pommier qui vont m'aider sur ce point.

Bien loin d'être ironique, et ne pouvant en aucun cas être interprété de la même manière que les œuvres de Bronzino, malgré la présence d'un grotesque, le Portrait de Laurent de Médicis (fig. 5), commandité après la mort du prince dans un souci de légitimation dynastique, est un exemple d'une nouvelle conception du portrait « intellectuel » qui se met en place dans les années 1530. Dans une lettre de la même époque, Vasari explique en détail la signification complexe de son portrait ${ }^{43}$ : d'une part Laurent de Médicis est représenté, idéalisé; d'autre part, des éléments sont ajoutés autour de sa figure afin d'expliciter allégoriquement son histoire. Edouard Pommier voit dans ce portrait, ainsi que dans celui d'Alexandre de Médicis et les lettres qui les accompagnent, un tournant dans la pratique du portrait :

"Les deux lettres de Vasari nous font assister à la genèse et à la justification d'un nouveau genre de portraits : il ne s'agit pas du portrait avec un attribut, mais bien du portrait emblématique, c'est-à-dire du portrait dédoublé, entre la représentation au naturel, directe ou indirecte, d'un visage et d'une personne, et la disposition de divers éléments, qui sont en principe des objets, réels ou fictifs, mais qui peuvent être aussi un paysage et qui racontent une histoire, c'est-à-dire ont une relation avec le personnage représenté. Le portrait emblématique explicite donc, par des figurations extrinsèques au modèle, son caractère, sa biographie, ses actions c'est-à-dire soit des phénomènes invisibles, soit des phénomènes étalés dans un temps étranger à celui de l'élaboration $d u$ portrait. On entre dans un processus au bout duquel le portrait se transforme en un objet complexe à deux niveaux $»^{44}$. 
Fig. 5

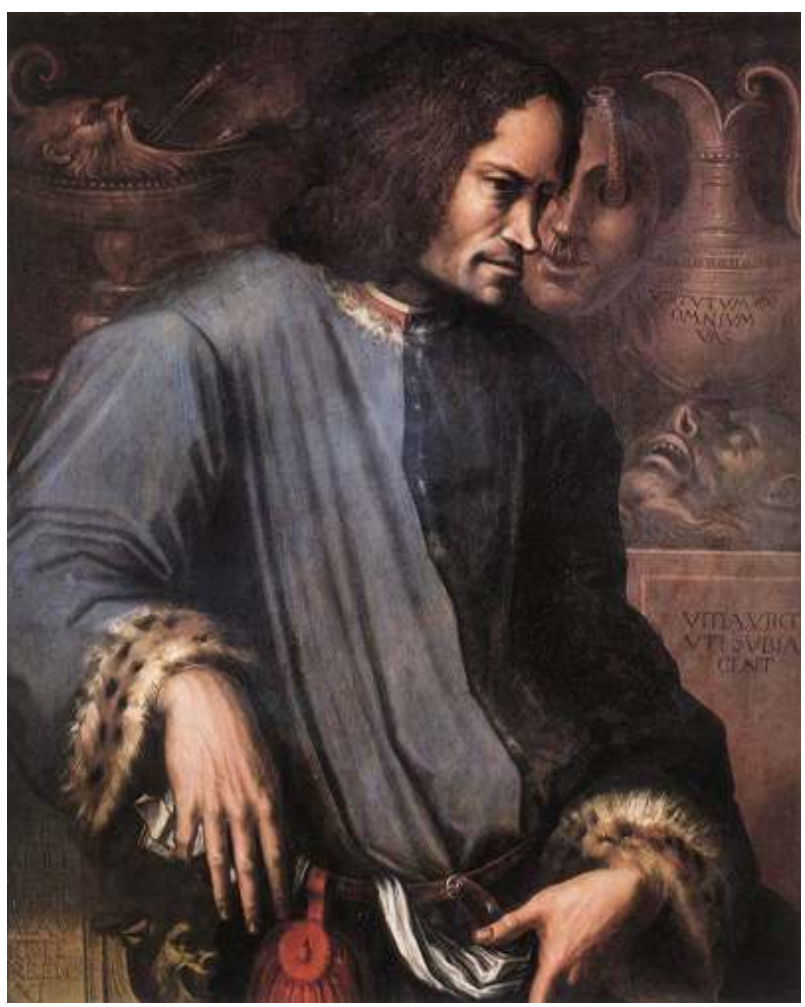

Giorgio Vasari, Portrait de Laurent le Magnifique, vers 1535, huile sur bois, $90 \times 72 \mathrm{~cm}$, Galleria degli Uffizi, Firenze. Avec l'aimable autorisation du Ministero dei Beni e le Attività Culturali.

www.polomuseale.firenze.it/uffizi

C'est précisément ce double niveau qu'utilise Bronzino, entre la représentation au naturel et la disposition d'un autre élément - et j'insiste sur le terme « disposition » -, en particulier un grotesque. Le portrait de Vasari indique qu'à Florence, vers 1535, les peintres pouvaient concevoir une œuvre qui mette en jeu ces deux niveaux.

Le rapprochement entre les œuvres de Bronzino et celles de Vasari doit néanmoins en rester là ${ }^{45}$. Alors que Bronzino représente des patriciens et des patriciennes, Vasari dépeint un prince illustre par le portrait duquel il désire se faire apprécier à la cour des Médicis. Il n'est donc pas question de glisser une quelconque ironie dans son œuvre. Et afin de supprimer toute ambiguïté quant à sa signification, Vasari fixe dans sa lettre très clairement le sens du grotesque en ces termes : «Il y aura une tête du Mensonge, feinte en marbre, qui se mord la langue, découverte par la main du Magnifique Laurent $»^{46}$. Le grotesque représente cette fois de manière univoque le vice, mais la nécessité qu'a pu ressentir Vasari d'expliciter et de fixer le sens de son œuvre montre que le grotesque pouvait être compris à son époque de manières variées.

\section{De l'ironie courtisane à l'ironie critique}

Il reste alors à comprendre pourquoi les personnages peints ont toléré - ou désiré - que soit glissée de l'ironie dans leurs portraits. Il est en effet ici évident que le peintre ne peut être considéré comme le seul responsable de cet effet; les commanditaires gardaient sans aucun doute un œil aiguisé sur des œuvres qui devaient exprimer la meilleure part d'eux-mêmes. Une telle ambiguïté dans la définition de l'identité n'est 
pas étonnante dans la Florence renaissante. Dans son étude sur le façonnement de l'identité dans la société florentine d'alors, Weissman met l'accent sur l'imbrication des différentes relations sociales dans lesquelles les Florentins étaient impliqués et par lesquelles la famille, les voisins, les amis, les clients et les patrons se mêlaient et se recoupaient. Une telle complexité sociale nécessitait de préserver des engagements sans en affaiblir d'autres. Par conséquent, «une des clés de l'interaction à la Renaissance est ce que l'on pourrait appeler l'importance d'être ambigu. Par cela, j'entends que la priorité était faite à un discours intelligent et mystérieux qui pouvait prendre différentes formes, du double entendre, de la métaphore, du flou au mensonge et à l'esquive directe ${ }^{47}$. Sans expliquer l'ironie des portraits de Bronzino et l'ambiguïté qui en découle, cette information permet de les replacer dans un contexte qui les rend historiquement plausibles.

31 Mais la raison du désir de paraître ironique à son propre sujet doit être recherchée ailleurs, en particulier dans les habitudes et les préceptes de conduite de l'époque. Sous l'autorité de Cosme Ier, les historiens l'ont assez répété, le portrait, notamment dynastique, devient un moyen de consolider le pouvoir absolu que le prince entend instaurer. A la même époque et lié à ce nouveau pouvoir accordé au genre, un certain élitisme commence à émerger: le portrait, puisqu'il glorifie son modèle, ne peut s'appliquer à n'importe qui. L'opinion de l'Arétin, exprimée dans une lettre à Leone Leoni en 1545, est exemplaire sur ce point: "Il ne faut pas croire que les lois des Anciens aient permis qu'on fasse des médailles de personnes qui n'étaient pas dignes. C'est ta honte, ô siècle, de tolérer que des tailleurs et des bouchers apparaissent vivants en peinture $»^{48}$.

32 Il ne s'agit pas ici de comparer le jeune homme et les dames de cour peints par Bronzino à des bouchers, mais bien d'insister sur l'autorité que pouvait leur octroyer le portrait. A une époque où le prince représentait, en particulier à travers ses portraits, le degré le plus haut de la hiérarchie, il ne fallait surtout pas risquer de se surestimer, et par conséquent de lui manquer de respect. Le courtisan devait en effet être «modeste et plein de retenue, usant toujours, et surtout en public, de la révérence et $\mathrm{du}$ respect qui sont convenables au serviteur envers son seigneur $\aleph^{49}$. Les modèles de Bronzino ont ainsi pu souhaiter marquer, par une ironie courtisane, leur modestie. En se présentant comme conscient de la noblesse que leur pose suggère tout en la relativisant, le jeune homme et les dames de cour envoient un double message tout à fait adapté au comportement qu'ils doivent adopter. D'une part, ils se mettent en valeur en insistant, par comparaison aux mascarons, sur leur grâce, leur beauté et leur vertu, mais aussi en affirmant leur adhésion à la mode esthétique et à l'humour notamment littéraire de leur temps. D'autre part, et dans la même représentation, ils se montrent modestes par une auto-ironie amusante, légère et totalement adaptée à l'humour et à l'esthétique de l'époque. L'ambiguïté interprétative de ces portraits paraît comme une réponse exacte à la mince marge de manœuvre laissée au courtisan par le Federico Fregoso du livre de Castiglione : «J'estime en outre que de même qu'il est mal de chercher une fausse gloire dans ce qu'on ne mérite point, de même il est mal de se frustrer soi-même de l'honneur dû et de ne pas chercher cette louange qui est la vraie récompense des entreprises vertueuses $»^{50}$.

33 L'ironie qui se manifeste dans les portraits de Bronzino n'est donc pas subversive, mais au contraire soumise à l'autorité. C'est une ironie légère qui ne propose pas de critique, si ce n'est une auto-critique de parade. Elle est pour ainsi dire légitime. Deux siècles 
plus tard, vers 1750 , une ironie beaucoup plus piquante et acerbe se manifestera, à travers le même mécanisme, dans un portrait de Tiepolo, par ailleurs connu pour son humour et son ironie visuels ${ }^{51}$. Dans son Portrait de procureur (fig. 6), l'artiste propose exactement le même dispositif de représentation. Dans l'impossibilité d'effectuer ici une analyse complète de l'œuvre, il est néanmoins important de constater la continuité du mécanisme visuel de l'ironie tout en notant l'évolution de sa nature et de son message en fonction du contexte. A nouveau, le représenté évoque la valeur et le pouvoir du modèle. A nouveau, le dispositif représentatif, en juxtaposant un visage sculpté au-dessus de la tête du modèle, lui oppose un commentaire ironique. Mais deux changements interviennent, qui modifient la nature de l'ironie. D'abord, les mascarons amusants sont remplacés chez Tiepolo par une figure animale plus effrayante. Celle-ci, en outre, domine le procureur, alors que les mascarons étaient visuellement inférieurs aux modèles de Bronzino. Ce changement dans la composition marque également une évolution dans la visée critique de l'ironie. Tiepolo ne propose pas, comme Bronzino, une ironie légère ; c'est cette fois un jugement subversif qui se joue. Si l'ambiguïté interprétative demeure, elle penche néanmoins au XVIII ${ }^{\mathrm{e}}$ siècle du côté de la laideur et du vice, qui dominent le procureur. Elle manifeste la réticence de Tiepolo face à une justice vénitienne fantoche, qu'il ne s'est pas privé de caricaturer à plusieurs reprises (fig. 7).

Fig. 6

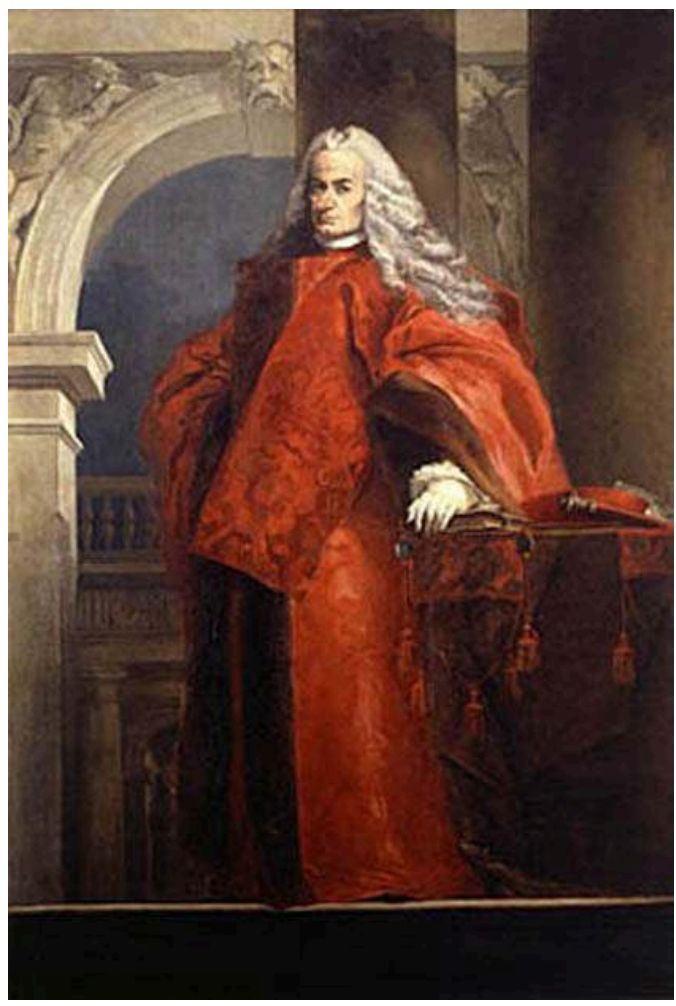

Giovanni Battista Tiepolo, Portrait du procureur Dolfin, vers 1750, 235 x 158 cm, Museo della Fondazione Querini Stampalia, Venezia. Avec l'aimable autorisation du musée.

www.querinistampalia.it 
Fig. 7

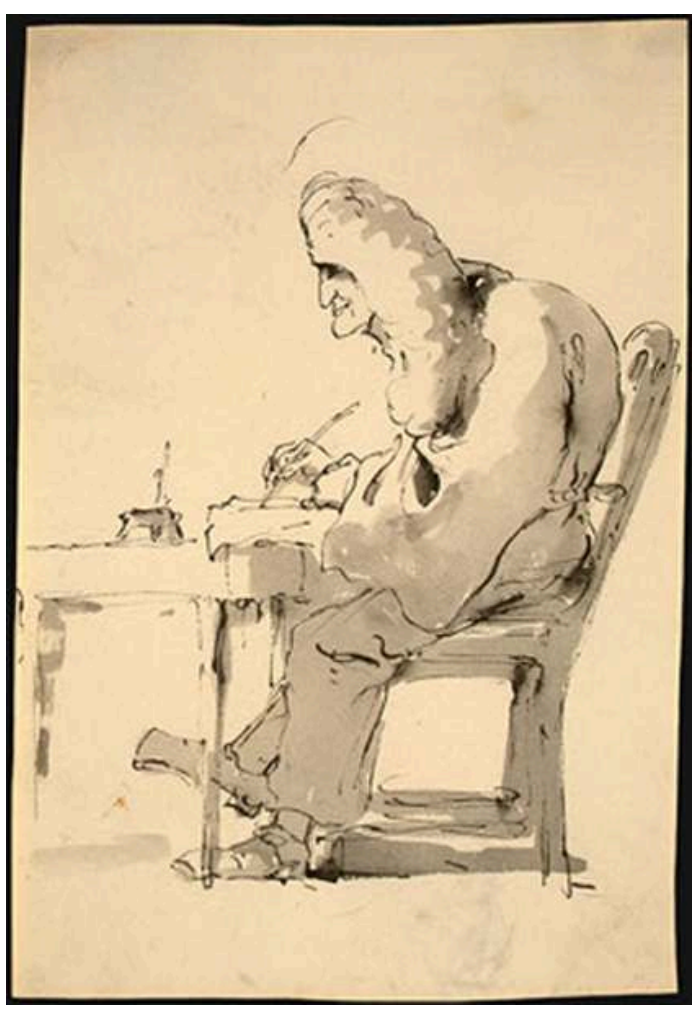

Giovanni Battista Tiepolo, Avocat vénitien à son bureau, vers 1760, plume et encre avec lavis gris sur papier verger, 20,5 × $14 \mathrm{~cm}$, National Gallery, Washington. Avec l'aimable autorisation du musée.

www.nga.gov

\section{La représentation prise pour cible?}

Si l'ironie s'est sans doute manifestée dans des portraits postérieurs à Bronzino (mais il ne faut pas en déduire qu'il ait eu une influence particulière dans ce domaine), il est beaucoup moins aisé d'en trouver avant lui dans le genre du portrait. Nous avons vu, notamment avec Edouard Pommier, qu'à partir du deuxième tiers du $\mathrm{XVI}^{\mathrm{e}}$ siècle florentin émerge une nouvelle conception du genre qui autorise un dispositif figuratif ironique. Sans faire ici de Bronzino le premier portraitiste ironiste, l'hypothèse peut être posée - certes précaire encore - d'un début d'ironie picturale dans le portrait - et seulement dans ce genre - qui s'ancrerait dans cette période particulière de l'histoire de l'art. Il n'est en effet pas étonnant que l'ironie trouve sa place à l'époque du maniérisme, qui manie avec agilité la citation, la référence et le détournement d'œuvres antérieures. Et la crise de confiance qu'éprouvent les artistes maniéristes, en particulier florentins, à l'égard de la représentation classique telle qu'elle se définit au cours du XV $\mathrm{XV}^{\mathrm{e}}$ siècle doit aussi être prise en compte dans la compréhension des portraits de Bronzino. L'ironie étudiée jusqu'à présent prenait pour cible, sans critique subversive, les modèles eux-mêmes. Il se pourrait également (je ne résiste pas à la tentation d'évoquer cette hypothèse) qu'elle se porte sur la représentation elle-même. La Haute Renaissance avait concilié sans aucun problème les deux exigences fondamentales de la représentation, et en particulier du portrait: l'imitation de la 
réalité ne semblait pas être opposée à l'idéalisation des formes. Erwin Panofsky a montré comment la période qui suit ne parvient plus à harmoniser cette contradiction :

"Cette opposition existait déjà lorsqu'on exhortait l'artiste à embellir les données de la réalité et qu'on exigeait par ailleurs qu'il fît preuve, jusqu'à l'illusion, de fidélité à la nature. Ce qui est nouveau, c'est plutôt la conscience que l'on prend de l'opposition qui existe entre ces deux « postulats » relatifs à l'amélioration ou à l'imitation du réel. La logique de l'ancienne peinture, qui admettait simultanément les deux postulats, se métamorphose ainsi en une logique nouvelle qui impose de choisir l'un ou l'autre ${ }^{52}$.

$36 \mathrm{Au}$ milieu du $\mathrm{XVI}^{\mathrm{e}}$ siècle, un malaise résulte inévitablement de cette nouvelle conscience de l'incompatibilité entre ressemblance et idéalisation. Le genre du portrait en cristallise toute la contradiction, dans la mesure où l'autorité et le pouvoir que confère le portrait nécessitent à la fois que l'on reconnaisse le modèle et qu'on l'admire. Bronzino, membre de l'Accademia Fiorentina pendant plusieurs années, grand connaisseur de Pétrarque et de Dante, comme en témoigne sa production littéraire, était un homme cultivé ; on peut lui prêter, si ce n'est une participation, du moins une connaissance des débats artistiques de son époque. Admirateur de Michel-Ange, il ne pouvait ignorer que son prédécesseur avait pour sa part renoncé à la ressemblance dans le travail du portrait, en particulier dans ceux de Julien et de Laurent de Médicis à la sacristie de San Lorenzo ${ }^{53}$. Il ne fait enfin aucun doute que Bronzino ait lui-même été confronté à la contradiction pratique entre ressemblance et idéalisation lors de l'exécution de ses portraits. Vasari, en affirmant que Bronzino « fit le portrait du duc à quarante ans et celui de la duchesse, tous deux aussi ressemblants que possible $"^{54}$, voulaitil exprimer ce tiraillement? Celui-ci se perçoit du moins dans l'idéalisation individualisée des quatre portraits qui nous intéressent. Ils présentent en effet tous, mais dans une moindre mesure pour celui de Turin, une géométrisation du visage qui ne pouvait être la copie exacte du modèle, mais qui devait rester liée aux particularités de chaque commanditaire. Les examens de laboratoire nous apprennent par ailleurs que le peintre a retouché à de nombreuses reprises le visage du jeune homme au livre, au point que le résultat final semble très différent du visage initial ${ }^{55}$.

De ces différents éléments résulte une compréhension nouvelle des grotesques de Bronzino. Il ne semble pas exclu que l'ironie, pour le dire de manière très schématique, n'exprime pas uniquement "je suis un courtisan ou une dame de cour très noble" avec une pointe d'ironie, mais également "je suis un portrait" avec une dérision qui indique l'impossibilité et la contradiction alors inhérente à cette pratique. En effet, dans son niveau transitif, qui donne à voir une part de l'histoire sur le mode de la fenêtre albertienne, la peinture présente un portrait établi selon les règles classiques de la représentation, c'est-à-dire dans une construction perspective et dans l'imitation bien qu'idéalisée de la nature. Ce niveau transitif repose sur les valeurs classiques de la représentation : un portrait est ressemblant tout en montrant la noblesse du modèle, c'est-à-dire en l'idéalisant. L'illusion est si bien réussie que le spectateur croit être face au modèle et en oublie le peintre. En revanche, par le grotesque, ce dernier attire l'attention sur l'incohérence et le paradoxe de cette illusion et de cette transparence des formes. André Chastel a relevé l'opposition totale que constitue le grotesque par rapport aux règles de la représentation classique. En effet, les artistes

« ont compris d'emblée la singularité et l'intérêt de ce système décoratif inédit, ou plus oublié. La portée en est considérable à nos yeux parce que, sous le couvert de l'antique, on tient là un principe de style exactement inverse de ce qu'exige et fonde au même 
moment l'ordre classique. On peut en énoncer l'originalité à l'aide de deux lois, qui faisaient et font toujours le charme irrésistible des grottesques : la négation de l'espace et la fusion des espèces, l'apesanteur des formes et la prolifération insolente des hybrides. [...] Le domaine des grottesques est donc assez exactement l'antithèse de celui de la représentation, dont les normes étaient définies par la vision «perspective » de l'espace et la distinction, la caractérisation des types ${ }^{56}$.

En opposant ainsi des grotesques aux visages des modèles, et en les liant comme nous l'avons vu de manière très intriquée, le peintre répond à des normes de représentation opposées aux règles classiques de la représentation mises en œuvre dans le reste du tableau.

\section{La perspective de l'ironie}

Observer une œuvre sous l'angle de l'ironie, ce n'est pas nécessairement contester les analyses qui en ont déjà été proposées. C'est envisager qu'elles peuvent s'enrichir d'une compréhension qui puise ses ressources dans une ambiguïté interprétative souvent condamnées à être ignorée. Cette interprétation renouvelée de l'œuvre n'a pas pour seul intérêt de revisiter l'œuvre elle-même ; elle engage aussi l'historien à mobiliser des informations sur lesquelles il ne s'arrêterait sans doute pas sans une interrogation sur l'ironie. Dans les portraits de Bronzino, cette interrogation ne permet pas seulement de considérer les grotesques sous tous leurs aspects pour en extraire le double sens, et la mise à distance du premier degré sérieux qu'ils instituent; elle oblige également à considérer les normes et les habitudes de conduite de l'époque selon un point de vue particulier. L'ambiguïté dans la définition de l'identité à la Renaissance, l'importance de la modestie du courtisan par rapport à son prince, la place que l'ironie prenait dans les règles de bienséance édictées par Castiglione ${ }^{57}$, la redécouverte à cette époque de l'ironie socratique, l'utilisation particulière du portrait - dont le pouvoir symbolique gagnait en importance - sont des éléments que l'analyse de l'ironie a rendu pertinents. La perspective de l'ironie peut donc espérer attirer l'attention sur des composantes historiques qui, dans d'autres circonstances, n'apparaitraient pas comme significatives. Passer à côté de l'ironie constitue une sous-interprétation sans doute aussi dommageable que la surinterprétation qui consiste à en trouver là où il n'y en a pas. S'il faut garder à l'esprit que l'ironie reste sans doute un phénomène minoritaire dans l'histoire de la peinture, il vaut la peine, lorsque cela se justifie, de l'interroger comme telle afin d'ouvrir la voie à une compréhension plus stable de son dispositif figuratif.

41 Cet objectif n'est en aucun cas atteint aujourd'hui. Et pourtant les portraits de Bronzino ont permis de mettre le doigt sur quelques éléments selon moi cruciaux. J'aimerais d'abord insister sur la nécessité d'aborder l'ironie picturale en termes de codes de représentation et donc de l'envisager, dans un premier temps du moins, dans un genre particulier. Le cas de Bronzino a en effet montré l'utilité de concevoir le phénomène dans le cadre des codes de représentation du genre particulier du portrait au XVI ${ }^{\mathrm{e}}$ siècle. L'attachement particulier au portrait permet en outre d'appréhender l'ironie comme une catégorie à part entière, et qui ne serait pas constituée d'éléments hybrides ramenés sans réelle justification dans un entre-deux mal défini. L'ironie n'est pas la caricature, elle n'est pas la satire non plus; ces deux genres ne proposant qu'un seul sens et non une superposition ambiguë de significations. L'ironie n'est pas non plus 
l'allégorie qui, bien qu'ayant plusieurs sens, ne les confronte pas de manière conflictuelle.

Cette précision méthodologique confirme le recours prudent qu'il convient de faire à la théorie sémiologique et linguistique, qui ne donne pour sa part pas d'importance au genre dans lequel l'ironie se manifeste. Les acquis théoriques sur l'ironie discursive sont pourtant indispensables, parce qu'ils nous aident dans un premier temps à repérer le phénomène. Ce sont en effet les principes généraux proposés par Oswald Ducrot qui ont permis d'élaborer une description, encore en évolution, de l'ironie figurative. Elle apparaît pour l'instant comme la superposition de plusieurs significations en concurrence, toutes visibles dans le tableau : le premier degré transparent, perceptible dans la profondeur de la troisième dimension, est relativisé lorsque l'on remonte, pour ainsi dire, au deuxième degré qui n'apparaît qu'à la surface de la toile, d'abord invisible, pour en percevoir la représentation du représenté. Une autre œuvre de Bronzino, mais qui mériterait des paragraphes entiers, permet de supposer que ce conflit entre représenté et représentation pourrait, d'une part, avoir une valeur assez générale, et d'autre part dépasser les limites du portrait. Le tableau de Vénus et Cupidon, de National Gallery de Londres, ne mêle-t-il pas ironiquement - dans la transitivité du représenté la volupté féminine trompeuse, et - dans l'opacité de la peinture et la représentation une touche sensuelle et une mise en valeur de la peau de Vénus qui s'inscrit précisément en faux par rapport à cette moralité austère ?

\section{NOTES}

1. Cf. Paul Barolsky, Infinite Jest, Wit and Humor in Italian Renaissance Art, Columbia, University of Missouri Press, 1978; Ernest B. Gilman, The Curious Perspective: Literary and Pictorial Wit in the Seventeenth Century, New Haven-London, Yale University Press, 1978, 267 p. ; David R. Smith, «Irony and Civility : Notes on the Convergence of Genre and Portraiture in Seventeenth-Century Dutch Painting ", dans Art Bulletin, vol. 69, n 3, 1987, p. 407-430; Leo Steinberg, "Steen's female gaze and other ironies ", dans Artibus et Historiae, n² 22, 1990, p. 107-128 ; Jörg Traeger, "Goyas königliche Familie : Hofkunst und Bürgerblick », dans Münchner Jahrbuch des bildenden Kunst, vol. 41, 1990, p. 147-181 ; James Elkins, "Uccello, Duchamp: the ends of wit ", dans Zeitschrift für Asthetik und allgemeine Kunstwissenschaft, vol. 36, 1991, p. 199-224; Philip Fehl, Decorum and Wit, the Poetry of Venetian Painting, Vienne, IRSA, 1992; Andrea Gottdang, "Die getauschte Erwartung: Witz und Ironie bei Giambattista Tiepolo », dans Artibus et Historiae, n 40, 1999, p. 151-168; Barbara Budnick, "Questions of irony in Pieter Bruegel's Magpie of the Gallows », dans Georges Bloch Jahrbuch des Kunsthistorischen Instituts der Universität Zürich, $\mathrm{n}^{\circ} 7,2000$, p. 68-83.

2. Barbara Budnick «Questions of irony in Pieter Bruegel's Magpie of the Gallows », op. cit., p. 70.

3. James Elkins, « Uccello, Duchamp: the ends of wit », op. cit., p. 214.

4. Dan Sperber, Deirndre Wilson, «Les ironies comme mentions », dans Poétique, n 36, 1978, p. 399-412.

5. Alain Berrendonner, Eléments de pragmatique linguistique, Paris, Editions de Minuit, 1981, p. 175.

6. Oswald Ducrot, Le Dire et le dit, Paris, Editions de Minuit, 1984, p. 183. 
7. Ibid., p. 211.

8. A propos des genres discursifs aux frontières de l'ironie, voir Pierre Schoentjes, Poétique de l'ironie, Paris, Seuil, 2001, p. 212-239.

9. Vladimir Jankelevitch, L'ironie, Paris, Flammarion, 2002, p. 132-133.

10. "The grotesques function like a wry half-smile musing over such a self-conscious display of elegance ». Deborah Parker, Bronzino, Renaissance painter as a poet, Cambridge, New York, Cambridge University Press, 2000, p. 159 (je traduis).

11. "These coarse and bizarre elements suggest the ironic self-awareness of the courtier who poses or postures so elegantly, both aware of their presence and aloof from them ». Paul Barolsky, Infinite Jest..., op. cit., p. 141 (je traduis).

12. Maurice Brock, Bronzino, Paris, Editions du Regard, 2002, p. 133.

13. Ibid., p. 10.

14. Le vêtement du jeune homme au livre répond par exemple à la recommandation de Castiglione : l'habit du courtisan doit toujours tendre « un peu plus vers le grave et le sérieux que vers le vain; c'est pourquoi il me semble que la couleur noire a meilleure grâce dans les vêtements que toute autre; et si toutefois elle n'est pas noire, au moins qu'elle tire sur le sombre ", Baldassare Castiglione, Le livre du courtisan, présenté et traduit de l'italien par Alain Pons, Paris, G. Lebovici, 1987, p. 140. Selon Maurice Brock, la position assise des femmes, qui répond à la forme du portrait conversationnel mis en place par Raphaël, est également liée aux règles de la vie courtisane, en particulier telles qu'elles sont édictées par Castiglione, Ibidem, p. 62-69.

15. Ce motif, redécouvert à la fin du 15ème siècle dans la Domus Aurea de Néron, avait très rapidement conquis les artistes florentins ainsi que leur commanditaires, que ce soit dans le domaine de la peinture ou de l'art décoratif. La famille Médicis manifeste notamment son goût pour ces éléments fantaisistes « à l'antique » dans un décor exécuté à San Lorenzo en 1532 par Giovanni da Udine, premier grand maître du genre, ainsi que dans sa commande à Marco Marchetti pour la décoration du Palazzo Vecchio que l'artiste exécute entre 1556 et 1557.

16. Alain Gruber, « Grotesques », dans Alain Gruber (dir.), L'art décoratif en Europe, Renaissance et Maniérisme, Paris, Editions Citadelles \& Mazenod, 1993, p. 193-273 (plus particulièrement pp. 209-210).

17. Cette forme d'humour avait été très largement remise à la mode par la cour des Médicis dans les productions artistiques et plus particulièrement littéraires. Malgré le pouvoir presque absolu qu'exerçait Cosme Ier, le rire avait pourtant sa place dans la Florence de son époque, comme l'explique Paul Barolsky dans son chapitre consacré à l'« aspect plus léger de la cour de Cosme de Médicis ». Francesco Berni (1497-1535) en particulier, fondateur de la maniera bernesca à la cour florentine, avait amené un renouveau d'humour et de satire dans la littérature toscane en parallèle aux productions lyriques inspirées de Pétrarque. La littérature de Bronzino en est un exemple important. "Il s'est toujours plu et se plaît encore à composer des vers. Il a écrit de nombreux capitoli et sonnets dont une partie sont imprimés. Sa poésie est surtout remarquable dans le genre des capitoli de Berni ", raconte Giorgio Vasari dans Vies des meilleurs peintres, sculpteurs et architectes, traduction et édition critique sous la direction d'André Chastel, Paris, Berger-Levrault, 1986, t. X, p. 207.

18. Philippe Morel, Les grotesques : les figures de l'imaginaire dans la peinture italienne de la fin de la Renaissance, Paris, Flammarion, 1997, p. 94.

19. Maurice Brock, Bronzino, op. cit., p. 71.

20. Ibid., p. 77.

21. Ibid., p. 72.

22. Ibid., p. 84-5.

23. Ibid., p. 135. 
24. Dans son analyse, Maurice Brock décrit les grotesques, mais n'explique pas les raisons de leurs traits particuliers, Ibid., p. 71, 77, 84, 132, 133.

25. Louis Marin, op.cit, p. 255. Selon Louis Marin: «Représenter signifie se présenter représentant quelque chose et toute représentation, tout signe ou procès représentationnel comprend une double dimension - dimension réflexive, se présenter; dimension transitive, représenter quelque chose - et un double effet : l'effet de sujet et l'effet d'objet. Sémanticiens et pragmaticiens contemporains les nommeront, et ce n'est point un hasard, "opacité" et "transparence" ». En d'autres termes, à son niveau transitif ou transparent, l'image montre, en feignant la fenêtre albertienne, une part de l'histoire et du réel. A ce niveau de transparence, le spectateur en oublie l'écran de la toile, le peintre et sa composition. Ce n'est qu'en redevenant conscient de ces éléments que le spectateur peut avoir accès au deuxième niveau, que Louis Marin qualifie de réflexif et d'opaque.

26. Philippe Morel, Les grotesques..., op. cit., p. 40-44.

27. Louis Marin, op. cit., p. 370. Chez Louis Marin, la notion de "réflexivité » fait surtout référence à la matérialité de l'œuvre que le niveau réflexif de la représentation révèle. Dans la problématique de l'ironie picturale, c'est moins la surface plastique de l'œuvre que l'intervention que le peintre y effectue et le dispositif représentatif qu'il y applique qui doit être pris en compte. 28. Daniel Arasse, Le détail, Paris, Flammarion, 1996, p. 373.

29. Maurice Brock, Bronzino, op. cit., p. 71.

30. L'époque à laquelle Bronzino réalise ses portraits ne connaît pas encore le genre de la caricature, dont on situe généralement l'apparition au début du XVIIe siècle. Au siècle précédant, les visages difformes et enlaidis, notamment réalisés par Léonard de Vinci, n'ont pas encore la particularité fondamentale de la caricature qui consiste à déformer les traits d'une personne réelle. Les grotesques de Bronzino constituent une étape intéressante qu'il s'agirait de replacer dans l'histoire de la caricature : ils donnent en effet aux grotesques, dont le genre est à l'époque répandu, un aspect chargé en les modelant à partir de visages réels.

31. Les recherches de Philippe Morel sur le grotesque suffisent à démontrer les fonctions extrêmement variées de cet élément à la Renaissance. Philippe Morel, Les grotesques, op. cit.

32. Vladimir Jankelevitch, L'ironie, op. cit., p. 62.

33. D'un point de vue philosophique, Anscombe estime qu'il faut renoncer à une analyse mentaliste de l'intention, qui consiste à « chercher quelque chose qui n'existe que dans la sphère de l'esprit ». G. E. M. Anscombe, L'intention, traduit de l'anglais par Mathieu Maurice et Cyrille Michon, Paris, Gallimard, 2002, p. 44. Elle veut favoriser une approche historique du phénomène, c'est-à-dire qui prenne en compte le contexte et les circonstances extérieures (pp. 81-95). Cette analyse historique de l'intention, selon Michael Baxandall, n'est précisément pas « raconter ce qui s'est passé dans l'esprit du peintre, mais construire une analyse susceptible de rendre compte des moyens dont il disposait et des fins qu'il poursuivait». Michael Baxandall, Formes de l'intention, traduit de l'anglais par Catherine Fraixe, Paris, Editions Jacqueline Chambon, 1991, p. 179.

34. Pierre Schoentjes insiste à plusieurs reprise sur la nécessité de connaître au mieux l'ironiste : «il importe [...] au plus haut degré de s'en faire une image la plus juste possible». Pierre Schoentjes, Poétique de l'ironie, op. cit., p. 147.

35. "The artist-poet does not choose one voice over another. Indeed, he often brings the two forms into playful contact. In Bronzino's work, lyric and burlesque, sacred and profane, sublimity and crudity coexist. To a lesser but still notable extent, Bronzino's painting displays the same dual creative tendencies. [...]Bronzino's simultaneous pursuit of two poetic forms suggests that it might be productive to think about his work less as being impenetrable and more as being pervaded by two forms of expression, one ordered and elegant, the other disordered and subversive ». Deborah Parker, Bronzino, Renaissance painter as a poet, op. cit., p. 168-169 (je traduis). 36. Philippe Morel, Les grotesques, op. cit., p. 94. 
37. Dilwyn Knox, Ironia. Medieval and Renaissance Ideas on Irony, Leiden, E. J. Brill, 1989; Norman Knox, The Word Irony and its Context, 1500-1755, Durham, Duke University Press, 1961.

38. Baldassare Castiglione, Le livre du courtisan, op. cit., p. 195.

39. Ibid., p. 194.

40. A ce sujet, voir Dilwyn Knox, op. cit., p. 110-126.

41. "L'autodépréciation ironique de Socrate prit la forme de l'ignorance feinte. Dans le Symposium de Platon, la comparaison qu'effectue Alcibiade entre Socrate et une statuette de Silène dérivait en partie de l'analogie entre la laideur du satyre, qui dissimule une image de dieu, et l'affirmation humoristique et ironique de son ignorance par Socrate, qui masque ses intentions sérieuses et vertueuses ». (" Socrates'ironic self-depreciation took the form of feigned ignorance. Alcibiades' comparison of Socrates to a Silenus statuette in Plato's Symposium derived partly from the analogy between the ugly, satyr statuette concealing an image of a god within and Socrates' humorous, ironic profession of ignorance masking his serious, virtuous intentions »), Dilwyn Knox, ibid., p. 111 (je traduis). Il est intéressant de noter ici l'ironie visuelle qui se manifeste, selon Alcibiade, dans la comparaison ironique entre un Socrate vertueux et une sculpture difforme: une comparaison de la même nature est effectuée entre les modèles des portraits de Bronzino et les grotesques.

42. Baldassare Castiglione, op. cit., p. 167.

43. Giorgio Vasari, «Lettere ", dans Paola Barocchi (dir.), Scritti d'arte del Cinquecento, MilaneNaples, Riccardo Ricciardi Editore, 1977, t. III, pp. 2707-2709.

44. Edouard Pommier, Théories du portrait de la Renaissance aux Lumières, Paris, Gallimard, 1998, p. 90-91 (je souligne).

45. Il faut aussi noter que les artistes se sont peut-être tous deux inspirés, mais chacun à leur manière, du Tombeau de Julien de Médicis, effectué par Michel-Ange à la Nouvelle Sacristie de San Lorenzo à Florence dans les années 1520. Plusieurs grotesques sont en effet incorporés à cet ensemble.

46. «Et in detto pilastro vi sarà una testa di una Bugia, finta di marmo, che si morde la lingua, scoperta dalla mano del Magnifico Lorenzo ». Giorgio Vasari, «Lettere », dans Paola Barocchi (dir.), Scritti d'arte..., op. cit., p. 2708 (je traduis).

47. "One key to the Renaissance etiquette of interaction is what one might call the importance of being ambiguous. By this I mean that high premium was placed on clever, cryptic speech that could take any form, from double entendre, metaphor, and vagueness, to outright lying and evasion ", E. Weissman, « The Importance of Being Ambiguous: Social, Relations, Individualism, and Identity in Renaissance Florence », dans Susanne Zimmerman et Ronald F. E. Weissman (dir.), Urban Life in the Renaissance, Londres - Toronto, Associated University Presses, 1989, p. 269-80 (p. 272 , je traduis).

48. Citée par Edouard Pommier, op. cit., p. 128.

49. Baldassare Castiglione, Le livre du courtisan, op. cit., p. 129.

50. Ibid., p. 116. Il me semble ici nécessaire de signaler une remarque qui m'a été faite par Anca Oroveanu au sujet des raisons des modèles de paraître ironiques. N'en ayant pris connaissance que quelques jours avant la publication de cet article, je la livre ici de manière brute sans pouvoir l'intégrer pleinement dans mon raisonnement. Selon elle, la fonction ironique des grotesques se situerait dans la complicité amusée qu'ils créent avec le spectateur plus que dans l'autodérision modeste des modèles. Cette suggestion me semble judicieuse, sans pour autant qu'elle s'oppose nécessairement à l'explication donnée plus haut. Les portraits étant réalisés dans un intervalle de quinze à vingt ans, il serait par ailleurs intéressant de pouvoir nuancer le propos en fonction de chacun d'entre eux ; cette réflexion est malheureusement pour l'instant freinée par le manque de sources ou la difficulté à y avoir accès.

51. Cf. Andrea Gottdang, op. cit.

52. Erwin Panofsky, Idea, traduit de l'allemand par Henri Joly, Paris, Gallimard, 1989, p. 101. 
53. Michel-Ange affirme en effet dans une lettre de 1544 son attention portée à la grandeur des figures bien plus qu'à leur ressemblance avec les modèles, Edouard Pommier, op. cit., p. 137.

54. Giorgio Vasari, op. cit., p. 206. Je souligne.

55. Maurice Brock, op. cit., p. 110-111.

56. André Chastel, La grottesque, Paris, Le Promeneur, 1988, p. 25.

57. Selon Castiglione, l'ironie permet de faire rire et d' "orner " la conversation courtisane : «Voyez combien les mots pris dans un sens contraire à celui qu'on attend donnent d'ornement, quand une clausule contraire s'oppose. " Castiglione, op. cit., p. 187. En plus de constituer un ressort comique, l'ironie peut également exprimer le sérieux, en particulier lorsque le blâme se manifeste par la louange: "Vous devez savoir aussi que des lieux d'où l'on tire des mots pour rire, on peut semblablement tirer des sentences graves pour louer et pour blâmer, et quelquefois avec les mêmes termes. [...] On dit encore : "c'est une dame qui n'a pas de prix", quand on veut la louer de sa prudence et de sa bonté ; on pourrait dire la même chose si on voulait la blâmer, en faisant allusion au haut prix que coûtent ses faveurs. » Ibid., p. 168.

\section{RÉSUMÉS}

Quatre portraits peints par Bronzino entre 1530 et 1555 sont ici analysés afin de dégager quelques caractéristiques de l'ironie picturale. Souvent classées par les historiens dans les registres soit comique, soit sérieux, ces peintures n'ont jamais été appréhendées dans leur polyphonie ironique. Or, des grotesques qui ornent chacun des portraits, se dégagent, par leur personnalisation adaptée à chaque modèle, plusieurs sens opposés et néanmoins conjointement présents. En s'appuyant sur certains acquis linguistiques, tout en préservant les spécificités visuelles de l'image, cet article tente, dans un premier temps, de décrire le mécanisme de l'ironie picturale. Il s'intéresse dans un deuxième temps aux conditions sociales, historiques et biographiques qui ont permis la production de telles œuvres dans la Florence renaissante.

\section{AUTEUR}

\section{PAULINE MARTIN}

Pauline Martin est historienne de l'art et diplômée de l'Institut National du Patrimoine à Paris. Commissaire associée pour la Foundation for the Exhibition of Photography, à Paris, elle est actuellement engagée dans une thèse de doctorat qu'elle mène conjointement à l'Ecole des Hautes Etudes en Sciences Sociales à Paris et à l'Université de Lausanne en Suisse. 